\title{
Total-electron-yield current measurements for near-surface extended x-ray-absorption fine structure
}

\author{
A. Erbil, ${ }^{*}$ G. S. Cargill III, R. Frahm, ${ }^{\dagger}$ and R. F. Boehme \\ IBM Thomas J. Watson Research Center, P.O. Box 218, Yorktown Heights, New York 10598
}

(Received 20 July 1987)

\begin{abstract}
A total-electron-yield technique is described in which near-surface extended $\mathrm{x}$-ray-absorption fine-structure (EXAFS) data are obtained from direct measurements of specimen current. Experiments with several model systems - amorphous germanium and crystalline germanium, nickel, and cobalt; and arsenic ion implanted into silicon-demonstrate that this technique can reproduce EXAFS $\chi(k)$ functions obtained from transmission and fluorescence measurements. Experiments also reveal that EXAFS amplitudes from total-electron-yield data can be 5-10\% smaller than those from transmission measurements for samples where the very-near-surface structure, at depths of tens to hundreds of angstroms, differs from the bulk structure. Measurements with buried layers confirm that the sampling depth for this total-electron-yield technique is determined primarily by the penetration ranges of Auger electrons emitted from the absorbing atoms. For the model systems listed above, $L M M$ Auger electrons have ranges of hundreds of angstroms and $K L L$ Auger electrons have ranges of thousands of angstroms. Expressions are derived for the sampling depth for total-electron-yield EXAFS experiments. The total-electron-yield technique described here is particularly useful for studying impurities within a few thousand angstroms of the surface of single crystals, where Bragg diffraction complicates the use of fluorescence measurements.
\end{abstract}

\section{INTRODUCTION}

Extended $\mathrm{x}$-ray-absorption fine-structure (EXAFS) spectroscopy has become established as a valuable tool for the determination of atomic scale structure for many types of materials. ${ }^{1,2}$ The technique consists of analyzing the fine structure extending several hundred $\mathrm{eV}$ above atomic core-level absorption edges to extract interatomic spacings and coordination numbers. It is sometimes advantageous to make EXAFS measurements by indirect methods, rather than by measurements of incident and transmitted $x$-ray intensities to determine absorption, which require specimens of appropriate, uniform thickness. Indirect measurement techniques, including x-ray fluorescence, ${ }^{3}$ Auger electron yield, ${ }^{4,5}$ and total electron yield, ${ }^{5,6}$ have been used for dilute samples and for samples with elements of interest localized in near-surface regions.

Fluorescence detection involves radiative decay of the core holes created by $\mathrm{x}$-ray absorption, and it has advantages for measuring EXAFS from minority components in dilute systems. ${ }^{3}$ Auger and total-electron-yield detection methods, which involve nonradiative decay of the core holes, have much shorter probe depths and have been used mainly for thin-film and surface EXAFS experiments. ${ }^{4-16}$ It was shown that the concept of phase transferability ${ }^{5}$ can be applied for EXAFS spectra measured by different techniques, including Auger, totalelectron-yield, and absorption detection. However, in some cases significant differences in EXAFS amplitudes have been observed by these different detection techniques. ${ }^{6,9,12,15}$ These results have implications for the extraction of coordination numbers. Martens et al. ${ }^{6} \mathrm{ob}-$ served an overall reduction of the total-electron-yield signal compared to the absorption spectra for $\mathrm{Ni}, \mathrm{Ge}$, and $\mathrm{Cu}$, using an electron multiplier type of detector for the total-electron-yield current. Stöhr et al. ${ }^{9}$ suggested that the amplitude differences observed between Auger and total-electron-yield detection techniques were the result of contributions of inelastically scattered photoelectrons. Guo and denBoer ${ }^{12}$ and Lytle et al. ${ }^{15}$ used a gasflow electron detector ${ }^{10}$ to measure electron total yield EXAFS signals for $\mathrm{Ni}, \mathrm{Cu}, \mathrm{Fe}$, and $\mathrm{Cr} K$ edges. They observed considerable differences for $\mathrm{Ni}, \mathrm{Cu}$, and $\mathrm{Fe}$ between the amplitude values obtained from transmission measurements and the corresponding values from a gasflow detector. The range of subsurface sensitivity for total-electron-yield EXAFS has been estimated to be $1000 \AA$ from measurements for $\mathrm{Cu}$ ( $K$ edge), ${ }^{6}$ less than $390 \AA$ for $\mathrm{Al}_{2} \mathrm{O}_{3}$ (Al $K$ edge), ${ }^{8}$ and $700-1000 \AA$ for GaAs ( $K$ edge). ${ }^{16}$

The present paper describes a method for totalelectron-yield measurements ${ }^{11,13}$ which is particularly well suited for near-surface EXAFS from single-crystal samples, where Bragg diffraction often makes fluorescence measurements difficult or impossible. The method reported here detects the total yield of electrons from the sample by measuring the sample current. Following descriptions of the technique and its applications, comparisons of total-electron-yield and transmission or fluorescence EXAFS measurements are given, to demonstrate the reliability of total-electron-yield measurements, particularly in light of previously reported difficulties with EXAFS amplitudes from total-electronyield measurements. ${ }^{6,9,12,15}$ Measurements and calculations are then given concerning the range of subsurface sensitivity for total-electron-yield EXAFS measurements. Results from the present study are also compared with those of previous investigations. 


\section{DESCRIPTION OF MEASUREMENT METHODS}

The arrangement used for the total-electron-yield measurements is shown schematically in Fig. 1. All measurements were made at the Cornell High Energy Synchrotron Source (CHESS) on line C1. A two-crystal Si(111) monochromator selected the incident photon energy $E$. The beam size at the sample was approximately $7 \times 1 \mathrm{~mm}^{2}$. The incident beam intensity $I_{0}(E)$ was monitored with an ion chamber.

The specimen chamber for total-electron-yield measurements consisted of a metal chamber with Kapton windows for incident and exit beams. It was evacuated to $30-50 \mu \mathrm{m} \mathrm{Hg}$ with a roughing pump. The specimen was electrically isolated from the chamber, but it was connected by a shielded cable to the input of a Keithley 427 current amplifier. For convenience in changing specimens, each specimen was glued with silver epoxy to a stainless-steel or aluminum frame. These frames were inserted into a Teflon sample stage. A spring wire made electrical contact to the metal frame.

EXAFS measurements made in this way, using specimen current to monitor total electron yield, avoid the problems with strong Bragg peaks which often complicate fluorescence EXAFS measurements. In EXAFS measurements on a single-crystal sample, diffraction occurs for incident photon energies which satisfy the Bragg condition for a set of planes of the sample. In fluorescence EXAFS measurements the diffracted beams directed toward the detector for some incident photon energies are often much more intense than fluorescence from the element of interest, so that the diffracted beams are not fully removed with the filter technique described by Stern and Heald. ${ }^{17}$ In fluorescence EXAFS measurements, this causes sharp peaks of varying strength to be superimposed on the EXAFS data at particular incident photon energies. Since the total-electron-yield EXAFS technique employs specimen current measurements rather than fluorescence intensity measurements, totalelectron-yield EXAFS are not directly affected by Bragg



FIG. 1. Arrangement used for total-electron-yield measurements. diffraction. However, weaker "glitches" were often present in the total-electron-yield EXAFS. They resulted from dynamical diffraction effects. ${ }^{18-20}$ For example, with As-in-Si single-crystal samples a small increase followed by a decrease occurs in the absorption by both the Si matrix and the As impurities whenever the incident photon energy, e.g., $x$-ray wavelength, passes through a diffraction condition for the particular orientation of the sample. Similar glitches were also sometimes seen in fluorescence EXAFS spectra even after the stronger Bragg peak features had been eliminated.

In the total-electron-yield experiments, dynamical diffraction glitches were eliminated by rocking the sample during the EXAFS measurements. Rocking by $\pm 5^{\circ}$ in each EXAFS scan served to smear out this type of glitch so that it was not visible in the final data. This was not effective for fluorescence EXAFS measurements, where effects of Bragg peaks are much greater than for total-electron-yield EXAFS measurements.

The rocking motion and capacitive coupling between the moving parts and the grounded walls of the chamber generated an ac current at the rocking frequency which was superimposed on the specimen current of interest. Measurements at each photon energy were taken over integral numbers of rocking periods, with a period of about $2 \mathrm{sec}$ and using a 6-sec/point measurement time, so the capacitive signal averaged to zero.

Effects on specimen current of positive and negative bias voltages were investigated by inserting 1.5-, 9-, and $90-\mathrm{V}$ batteries, in various combinations, between the specimen and the current amplifier. Total-electron-yield $K$-edge EXAFS measurements for a nickel foil sample were made for various different bias voltages. Applying bias greater than $\pm 1.5 \mathrm{~V}$ caused large drifts in the total specimen current but not in the edge-jump current, which is the change in specimen current which occurs when the incident photon energy is scanned from below to above the absorption edge [see Fig. 6(a)]. Biasing the sample negative with respect to the walls of the sample chamber increased the edge-jump current from its zerobias value by about $5 \%$ for $1.5 \mathrm{~V}$ and by $10 \%$ for 10.5 and $100.5 \mathrm{~V}$. Biasing the sample positive reduced the edge-jump current to $60 \%$ of its zero-bias value for 1.5 $\mathrm{V}$ and to $10 \%$ for $10.5 \mathrm{~V}$. These results indicate that the total-electron-yield current responsible for EXAFS consists mainly of ejected electrons with less than $1.5 \mathrm{eV}$ kinetic energy.

Increasing the positive bias to $100.5 \mathrm{~V}$ caused reversal of the sign of the edge-jump current, and this bias gave an edge-jump current amplitude about $40 \%$ of the zerobias value. This may be due to collection of electrons from residual gas ionization and electron avalanche events initiated by relatively small numbers of energetic $(6.5 \mathrm{kV}) K L L$ Auger electrons ejected from the nickel foil sample.

For the measurements described in this paper, the incident photon flux was approximately $10^{9} \mathrm{sec}^{-1}$ for energies near the $\mathrm{Ni}, \mathrm{Ge}$, and $\mathrm{As} K$-absorption edges. Total-electron-yield specimen currents $i_{\mathrm{TEY}}(E)$ were typically $10^{-12} \mathrm{~A}$.

An ion chamber was also placed after the specimen 
chamber to monitor the transmitted intensity $I(E)$. For transmission measurements, the monochromator was detuned to reduce the harmonic content of the incident beam. Fluorescence intensity $I_{F}(E)$ was measured for an As-in-Si sample with a scintillation counter and filter assembly ${ }^{17}$ placed $45^{\circ}$ above the incident beam and $2.5 \mathrm{~cm}$ from the sample. The major difficulty with the fluorescence measurements was the occurrence of Bragg diffraction maxima so intense that they were not fully removed by using the Ge filters. However, effects of Bragg peaks usually could be avoided by blocking them with lead tapes between the sample and the Ge filter.

\section{COMPARISON \\ OF TOTAL-ELECTRON-YIELD RESULTS WITH OTHER MEASUREMENTS}

This section describes comparisons of total-electronyield results with transmission and fluorescence EXAFS measurements. In some cases two measurements were made on the same specimen, e.g., total-electron-yield and transmission measurements for a $5-\mu \mathrm{m}$-thick foil of polycrystalline nickel. Simultaneous total-electron-yield and transmission measurements could be made for samples of appropriate and uniform thickness. However, it was found that the EXAFS $\chi(k)$ [see Eq. (2)] from such total-electron-yield measurements had an amplitude only about $75 \%$ that obtained from the transmission measurement. Most of this difference could be attributed to secondary electron signals coming from the back surface of the specimen, which combine with those from the specimen's front surface. As shown in Appendix A, including these back-surface signals suppresses the amplitude of the total-electron-yield EXAFS $\chi(k)$. Inserting a $100-\mu \mathrm{m}$-thick lead foil behind the specimen and in electrical contact with it eliminates this rear-surface current, producing a total-electron-yield EXAFS $\chi(k)$ in much better agreement with transmission results. Totalelectron-yield measurements $i_{\mathrm{TEY}}(E) / I_{0}(E)$ and transmission measurements $-\ln \left[I(E) / I_{0}(E)\right]$ are shown in Fig. 2 for the $5-\mu \mathrm{m}$ nickel foil.

In the discussions and equations which follow, $\mu(E)$ is used to represent $i_{\mathrm{TEY}}(E) / I_{0}(E)$ for total-electron-yield measurements, $-\ln \left[I(E) / I_{0}(E)\right]$ for transmission measurements, or $I_{F}(E) / I_{0}(E)$ for fluorescence measurements. For both transmission and total-electron-yield measurements of $\mu(E)$, linear preedge extrapolations $\mu_{\text {non- } K}(E)$, as shown in Fig. 2, were used to extract the $K$-absorption contributions $\mu_{K}(E)$,

$$
\mu_{K}(E)=\mu(E)-\mu_{\text {non- } K}(E) .
$$

For fluorescence measurements of As-in-Si, $\mu_{\text {non- } K}(E)$ was obtained from measurements on a silicon specimen containing no arsenic, with multiplicative scaling to match the As-in-Si fluorescence measurements in the preedge region.

Spline function fitting, to determine the $K$-shell background $\mu_{K, 0}(E)$, and normalization to the $K$-edge jump were used to obtain the EXAFS $\chi(k)$ function,

$$
\chi(k)=\frac{\mu_{K}(E)-\mu_{K, 0}(E)}{\mu_{K, 0}\left(E_{0}\right)},
$$
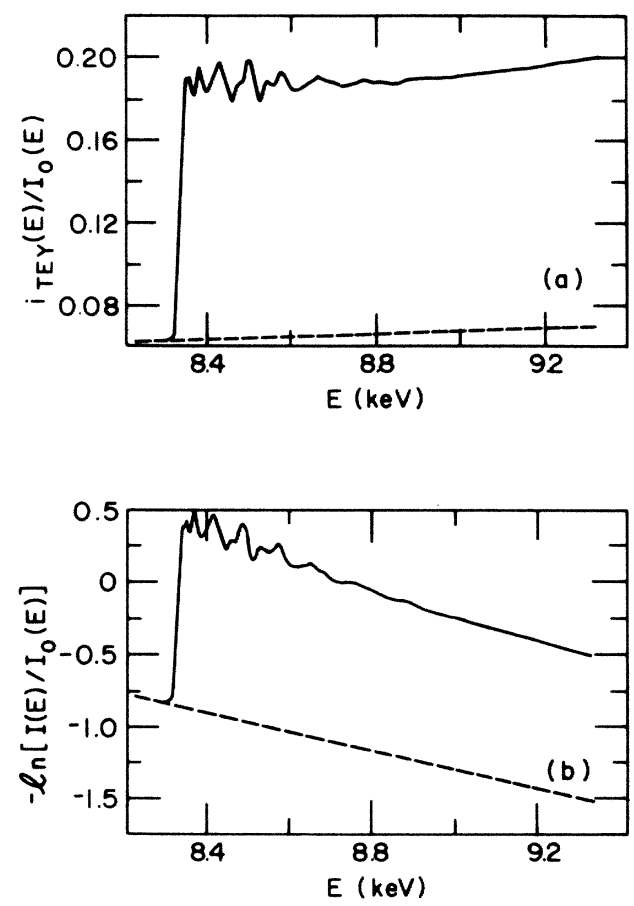

FIG. 2. Total-electron-yield and transmission measurements, (a) $i_{\mathrm{TEY}}(E) / I_{0}(E)$ and (b) $-\ln \left[I(E) / I_{0}(E)\right]$ for $5-\mu \mathrm{m}$ nickel foil, with rear-surface current eliminated. Vertical scales are arbitrary. Also shown are preedge extrapolations $\mu_{\text {non-K }}(E)$ (dashed lines).

where

$$
k=\left[2 m\left(E-E_{0}\right) / \hbar^{2}\right]^{1 / 2}
$$

and $E_{0}$ is the absorption edge energy.

\section{A. Photoelectron contributions in total-electron-yield measurements}

Using the edge jump $\mu_{K, 0}\left(E_{0}\right)$ in the denominator of Eq. (2) instead of $\mu_{K, 0}(E)$ was suggested by Stöhr et al. ${ }^{9}$ for total-electron-yield measurements, because of the way in which photoelectrons contribute to the totalelectron-yield current. This occurs mainly through secondary electrons caused by inelastic scattering of the photoelectrons. The number of secondary electrons produced by each photoelectron depends on the photoelectron's initial energy and is expected to grow with increasing $E>E_{0}$. Stöhr et al. ${ }^{9}$ have argued that this contribution should not contain EXAFS oscillations, because inelastic scattering keeps these photoelectrons from participating in coherent backscattering from near neighbors of the absorbing atoms. $\mathrm{Lee}^{4}$ had shown on theoretical grounds that the current from elastic photoelectrons should reproduce the EXAFS if the photoelectron collection is averaged over $4 \pi \mathrm{sr}$.

Stöhr et al. ${ }^{9}$ and others ${ }^{6}$ have argued persuasively that the contribution of elastic photoelectrons to totalelectron-yield current is very small compared with contributions of secondary electrons created by inelastic scattering of the Auger electrons or photoelectrons. It 
follows that dividing $\mu_{K}(E)-\mu_{K, 0}(E)$ by $\mu_{K, 0}(E)$ for total electron yield [see Eq. (2)] would give a $\chi(k)$ function which decreases in amplitude with increasing $k$ more rapidly than an equivalently defined $\chi(k)$ obtained from transmission measurements. However, dividing by $\mu_{K, 0}\left(E_{0}\right)$ rather than by $\mu_{K, 0}(E)$, as in Eq. (2), should give equivalent $\chi(k)$ functions for total-electron-yield and transmission measurements.

The relative contribution of photoelectrons, both elastic and inelastic, to the total-electron-yield current has not been quantified, in either experiment or theory. The extent to which secondary electron current from inelastic scattering of photoelectrons carries EXAFS oscillations also remains undetermined, apart from the negative conjecture by Stöhr et al. ${ }^{9}$ which has been questioned by Guo and denBoer. ${ }^{12}$ Indeed, it can be argued that whenever the inelastic scattering of a photoelectron occurs beyond the near-neighbor environment of the absorbing atom, the elastic backscattering from near neighbors, which is responsible for EXAFS phenomena, should be unaffected, and the flux of secondary electrons produced by these photoelectrons should contain EXAFS oscillations. Because of the overall energy dependence of this contribution to the total-electron-yield current, it could be responsible for differences in amplitudes between $\chi(k)$ functions from total-electron-yield and from absorption or fluorescence measurements, particularly at larger values of $k$. However, comparisons between total-electron-yield and transmission or fluorescence measurements for several model systems, discussed in Sec. III B, demonstrate that this type of photoelectron contribution to $\chi(k)$ must account for less than $10 \%$ of the total $\chi(k)$ up to $k=12 \AA^{-1}\left(E-E_{0} \approx 0.55 \mathrm{keV}\right)$ in these systems. However, numerical estimates of the relative contributions of photoelectrons and Auger electrons to the total-electron-yield current, given in Sec. IV F, show that secondary electrons produced by $0.5-\mathrm{keV}$ photoelectrons may be responsible for $10 \%$ of the totalelectron-yield current; these calculations do not address the question of whether this component of the current carries EXAFS oscillations, or whether it only contributes smoothly to the background. In the latter case, it would be eliminated from $\chi(k)$ by the edge-jump normalization and the subtraction of $\mu_{K, 0}(E)$ as in Eq. (2).

\section{B. Comparisons of EXAFS data}

Several previous studies had indicated total-electronyield amplitudes to be as much as $30 \%$ smaller than transmission amplitudes. ${ }^{6,9,12,15}$ Differences between total-electron-yield and transmission $\chi(k)$ amplitudes for our measurements were characterized by the multiplicative scaling constant $\kappa$ which yielded the best (leastsquares) agreement between $\chi_{\mathrm{TEY}}(k)$ and $\kappa \chi_{\text {trans }}(k)$. Results are given in Table $I$, and comparisons between total-electron-yield and transmission data are shown in Fig. 3 as $\chi_{\mathrm{TEY}}(k), \chi_{\text {trans }}(k)$, and $\Delta \chi=\chi_{\mathrm{TEY}}(k)-\chi_{\text {trans }}(k)$. A comparison of total-electron-yield and fluorescence results for an ion-implanted, laser-annealed As-in-Si sample is also included in Table $I$ and is shown in Fig. 3 as $\chi_{\mathrm{TEY}}(k), \chi_{\text {fluo }}(k)$, and $\Delta \chi(k)=\chi_{\mathrm{TEY}}(k)-\chi_{\text {fluo }}(k)$.
TABLE I. Samples used in comparisons of $\chi(k)$ between total-electron-yield and transmission or fluorescence measurements. $\kappa$ is the ratio between amplitudes of $\chi(k)$ for transmission or fluorescence measurements and for total-electron-yield (TEY) measurements, defined as described in the text.

\begin{tabular}{llc}
\hline \hline Sample & \multicolumn{1}{c}{ Description } & $\kappa$ \\
\hline $\mathrm{Ni}-1$ & $50-\mu \mathrm{m}$ foil, TEY & 1.03 \\
$\mathrm{Ni}-2$ & $100-\mu \mathrm{m}$ foil, TEY & 0.95 \\
$\mathrm{Ni}-3$ & $5-\mu \mathrm{m}$ foil, trans. and TEY & 0.88 \\
$\mathrm{Ni}-4$ & $\begin{array}{l}5-\mu \mathrm{m} \text { carbon-coated foil } \\
\text { trans. and TEY }\end{array}$ & 0.97 \\
$\mathrm{Co}$ & $\begin{array}{l}5-\mu \mathrm{m} \text { foil } \\
\text { trans. and TEY }\end{array}$ & \\
& 20- $\mu \mathrm{m}$ amorph. film & 0.95 \\
$\mathrm{Ge}-a$ & trans. and TEY & \\
& Crystal. powder, trans. \\
Ge- $c$ & single-crystal, TEY & 0.96 \\
& Ions implanted, laser-annealed \\
As-in-Si & fluo. and TEY & 1.01 \\
\hline \hline
\end{tabular}

Figure 4 shows detailed absorption features at the $\mathrm{Ni}$ $K$ edge, which are nearly identical in transmission measurements (sample $\mathrm{Ni}-3$ ) and in total-electron-yield measurements for sample Ni-1. However, differences are seen for total-electron-yield measurements for other samples (Ni-2 and $\mathrm{Ni}-3$ ), which are also the samples for which total-electron-yield measurements gave scaling constants $\kappa<1$. Both the different edge features and the reduced EXAFS amplitudes for these two samples probably arise from their having thicker surface oxides, in which the chemical and geometrical near-neighbor environment of $\mathrm{Ni}$ atoms is different than in the bulk. As noted in the next section, the total-electron-yield measurements contain a component which is particularly sensitive to the outer few hundred angstrom, very-nearsurface region. Some earlier reports of reduced amplitudes for total-electron-yield EXAFS compared with transmission EXAFS are probably of similar origin. Also, as noted above and in Appendix A, total-electronyield current from back surfaces of thin samples can also cause reduced amplitudes, and this may also be responsible for some previously reported difficulties.

Amplitudes for $\chi_{\mathrm{TEY}}(k)$ were further tested by making total-electron-yield measurements on the $5-\mu \mathrm{m}$ nickel foil sample after a carbon coating, approximately $500 \AA$ thick, had been evaporated onto the foil's exposed surface. This should eliminate the component of the totalelectron-yield current which has great very-near-surface sensitivity, as will be discussed in Sec. IV F. In fact, total-electron-yield results shown in Fig. 5 for this carbon-coated sample, $\mathrm{Ni}-4$, are nearly identical to the transmission measurements for the EXAFS region, with $\kappa=0.97$, and for the near-edge features (see also Fig. 4).

Our results, $\kappa=1.00 \pm 0.05$, for homogeneous samples indicate that $\chi(k)$ functions from total-electron-yield measurements agree well with $\chi(k)$ functions from transmission or fluorescence measurements. 

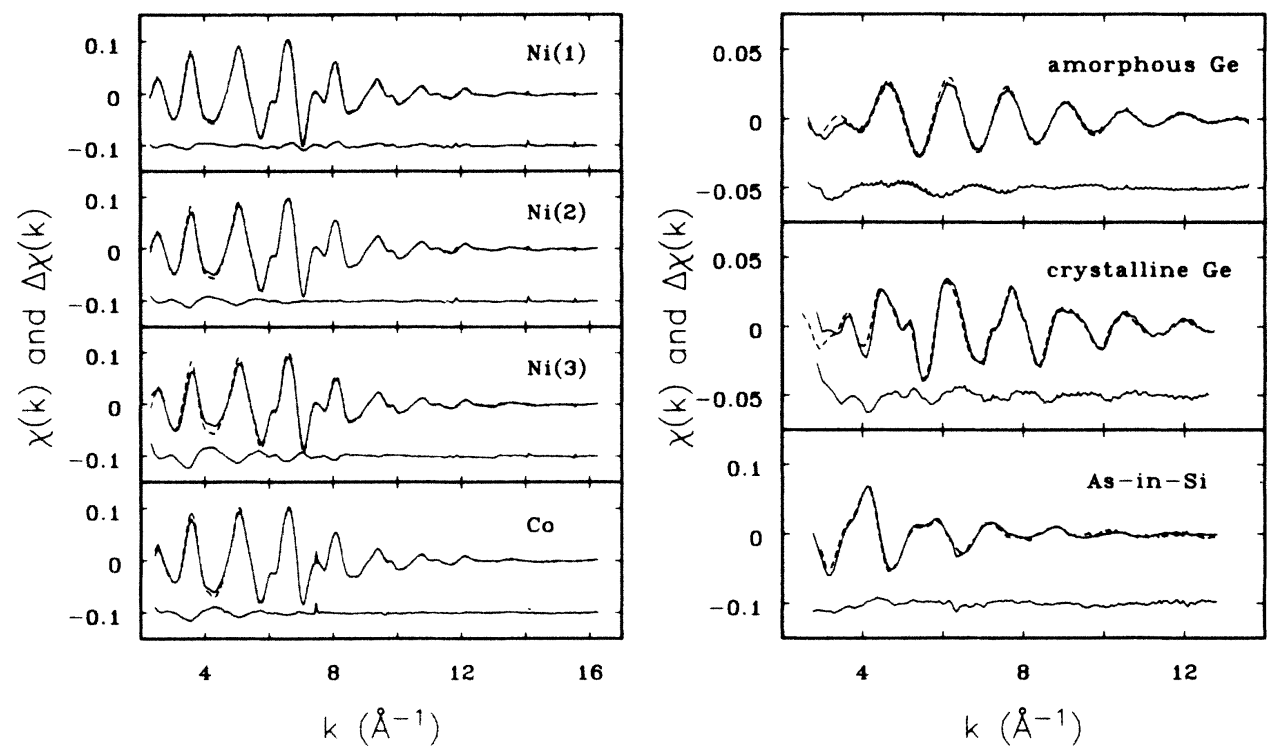

FIG. 3. Total-electron-yield (solid line) and transmission (dashed line) EXAFS data $\chi(k)$ for samples listed in Table I: Ni-1, Ni2, Ni-3, Co, amorphous Ge, and crystalline Ge. Also shown is a comparison of total electron yield and fluorescence (dashed line) for the As-in-Si sample listed in Table I. $\Delta \chi(k)$, the difference between the total-electron-yield and transmission or fluorescence data, is plotted displaced downward but in each case with the same vertical scale as $\chi(k)$.

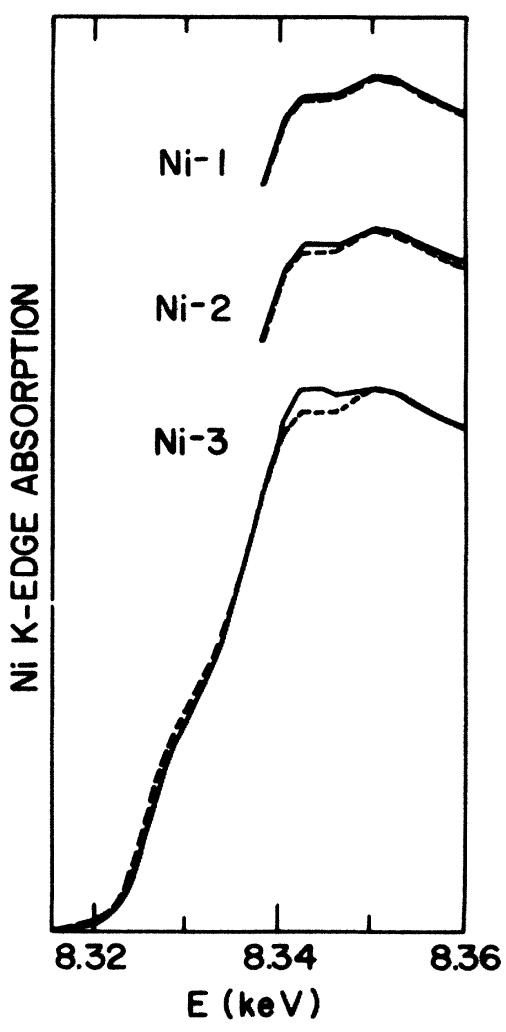

FIG. 4. Ni $K$-edge absorption features from transmission measurements (dashed line) for sample $\mathrm{Ni}-3$ and from totalelectron-yield measurements (solid line) for samples $\mathrm{Ni}-1, \mathrm{Ni}-2$, and Ni-3. Vertical scales and zeros have been adjusted to equate transmission and total-electron-yield data at 8.30 and $8.35 \mathrm{keV}$.

\section{SAMPLING DEPTH FOR TOTAL-ELECTRON-YIELD MEASUREMENTS}

\section{A. Experimental results}

Experiments were carried out to investigate the sampling depth for total-electron-yield EXAFS measurements. Specimens were prepared by sputtering approximately $100 \AA$ of $\mathrm{Ge}$ onto Si substrates, followed by evaporation of Si films of selected thicknesses $t_{\mathrm{Si}}$ up to 4000 $\AA$, as illustrated in Fig. 6(b). Total-electron-yield measurements were made in the vicinity of the Ge $K$ absorption edge $(11.10 \mathrm{keV})$ for each sample. Data for the sample with a $125-\AA$ silicon overlayer are shown in Fig. 6(a). Also shown are straight lines fitted to the data below the edge (11.0-11.07 keV) and above the edge $(11.15-11.40 \mathrm{keV})$ which were used to determine the time-integrated edge-jump current. Results from totalelectron-yield measurements for all of the samples, shown in Fig. 6(b), indicate that the edge jump decreases with increasing thickness of the silicon overlayer. For overlayer thicknesses of less than $500 \AA$ there is a sharper rate of decrease of edge jump with increasing overlayer thickness than for $500-4000-\AA$ overlayer thickness. There is significant edge-jump amplitude even for a 4000 - $\AA$-thick silicon overlayer. These data cannot be fitted with a simple exponential dependence of escape probability on overlayer thickness. The following discussion provides an explanation of the observed dependence on overlayer thickness and indicates that these total-electron-yield experiments have sampling depths determined primarily by the penetration range of Auger 
electrons, which is several thousand angstroms for $\mathrm{Ge}$ $K L L$ Auger electrons in silicon.

\section{B. Electron penetration range and production of secondary electrons}

The most important sources of total-electron-yield current associated with $K$-shell absorption for elements like $\mathrm{Co}, \mathrm{Ni}, \mathrm{Ge}$, and As are the Auger processes which result from nonradiative decay of the core hole produced by $x$-ray absorption. The situation is illustrated in Fig. 7. For elements ranging from cobalt $(Z=27)$ to arsenic $(Z=33)$, the most probable Auger processes are $K L L$ emission, with Auger electron energies of $6-9 \mathrm{keV}$, and $L M M$ emission, $0.7-1.2 \mathrm{keV} .{ }^{21}$ These Auger electrons lose energy to other (secondary) electrons, which in turn undergo inelastic scattering, to create additional secondary electrons.
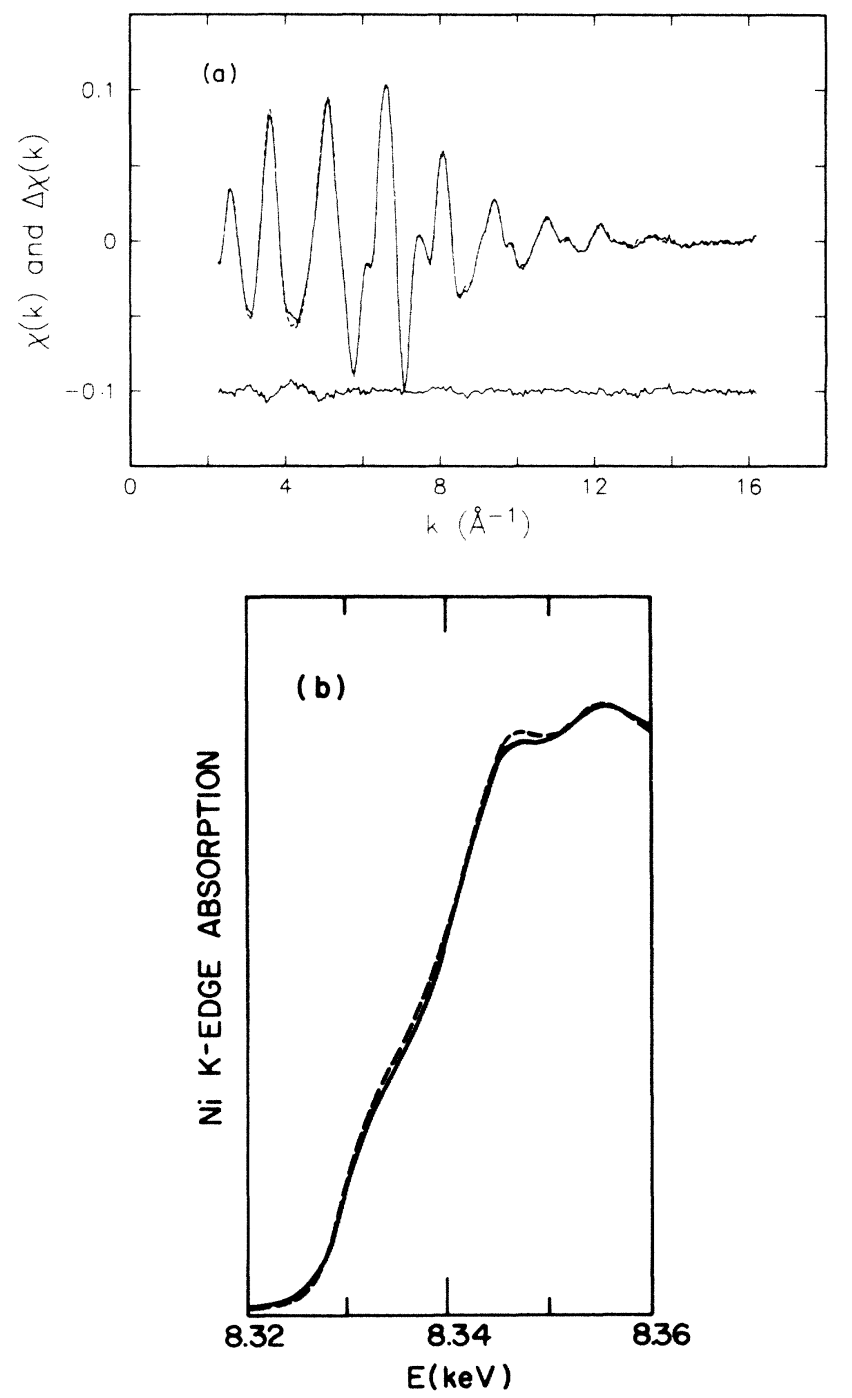

FIG. 5. (a) Total-electron-yield (solid line) and transmission (dashed line) EXAFS data, $\chi(k)$ and $\Delta \chi(k)$, for a carboncoated $5-\mu \mathrm{m}$-thick nickel foil (sample Ni-4). (b) Ni $K$-edge absorption features for total-electron-yield (solid line) and transmission (dashed line) measurements for this sample.
The sampling depth for total-electron-yield EXAFS measurements is determined mainly by the effective penetration range $R_{p}$ of the Auger electrons, which is the distance over which they travel and deposit their excess energy. Numerical values of $\boldsymbol{R}_{p}$ are needed to interpret results of the experiment described in the previous section and to predict whether the total-electron-yield technique will be useful for various types of samples. There are many reports of energy- and materialdependent electron penetration ranges measured using monoenergetic electrons; however, numerical results for a given energy and material differ by as much as a factor of 2 for different definitions of electron range and for different experimental methods. ${ }^{22-30}$ Within these limits, experimental results for the dependence of $R_{p}$ on initial electron energy $E$ of $1-10 \mathrm{keV}$ for various materials can be approximated by the empirical relationship ${ }^{29}$

$$
R_{p}^{(1)} \approx 1000 E^{1.4} / \rho
$$

with $R_{p}$ in angstroms and $E$ in $\mathrm{keV} ; \rho$ is the material's density in $\mathrm{g} / \mathrm{cm}^{3}$.

Other estimates of $\boldsymbol{R}_{p}$ have been obtained theoretical-
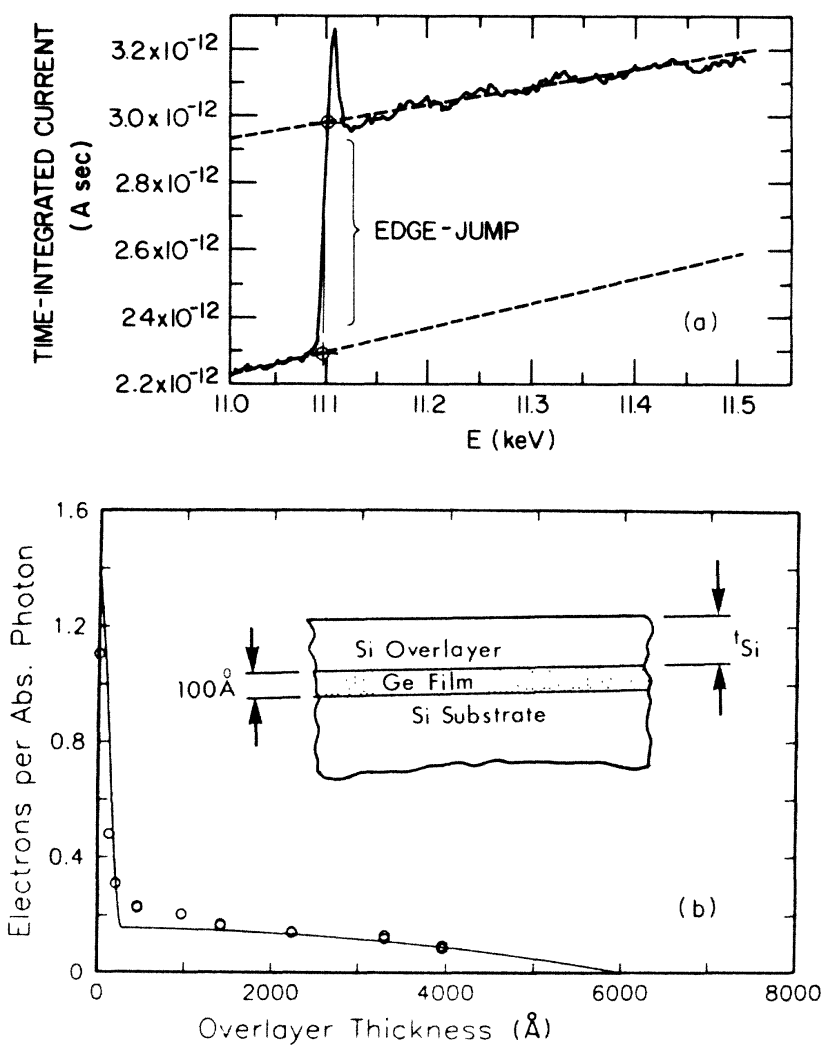

FIG. 6. Total-electron-yield measurements for Si-Ge-Si samples. (a) Data for the sample with a $125-\AA$ silicon overlayer, with straight lines fitted to the data and used to determine the time-integrated edge-jump current, as described in the text. (b) Edge jump, given as emitted electrons per photon absorbed in the Ge film, vs overlayer thickness $t_{\mathrm{Si}}$. Data points are from measurements and lines are from calculations for $K L L(8.6$ $\mathrm{keV})$ and $L M M(1.1 \mathrm{keV})$ Auger electrons, as described in the text. Inset: illustration of $\mathrm{Si}-\mathrm{Ge}-\mathrm{Si}$ samples. 
ly. Bethe's continuous slowing down approximation has been used to calculate the total path length traversed by an electron before it loses its excess kinetic energy, the Bethe range $R_{B}$. Tabulations have been published by Berger and Seltzer ${ }^{31}$ and by Sugiyama. ${ }^{32}$ This approximation has been used in Monte Carlo calculations of electron trajectories to obtain values of $R_{p} \cdot{ }^{29,33,34}$ The results of these calculations for initial electron energies of $5-10 \mathrm{keV}$ can be roughly approximated as

$$
R_{p}^{(2)} \approx R_{B} / 2
$$

Values of $R_{B}$ and of $R_{p}$ from Eqs. (4) and (5) are plotted for silicon in Fig. 8 and are given in Table II for $K L L$ and $L M M$ Auger electrons and for $0.5-\mathrm{keV}$ photoelectrons for various systems. $R_{p}^{(2)}$ values from Eq. (5) have been more useful than $R_{p}^{(1)}$ values from Eq. (4) for making accurate calculations of the depth sensitivity of total-electron-yield EXAFS measurements (see Sec. V). As shown in Table II, both $R_{B}$ and $R_{p}$ are much larger than estimates of the inelastic mean free path $R_{\text {inel }}$, which is the mean distance an electron travels before participating in an inelastic scattering event. ${ }^{35,36}$ This

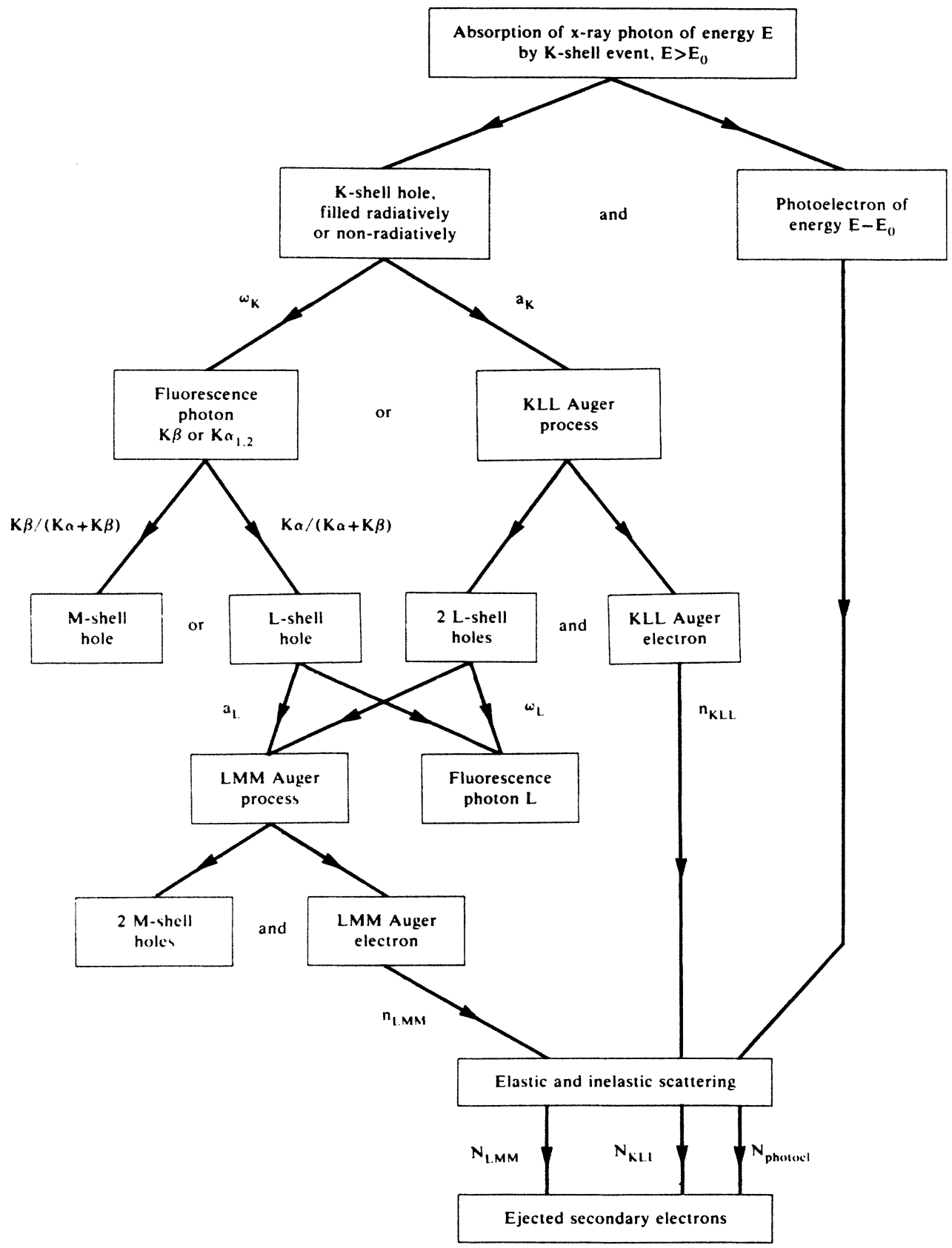

FIG. 7. Relationships among relevant processes in total-electron-yield current production, illustrated for the case of $K$-shell absorption. 


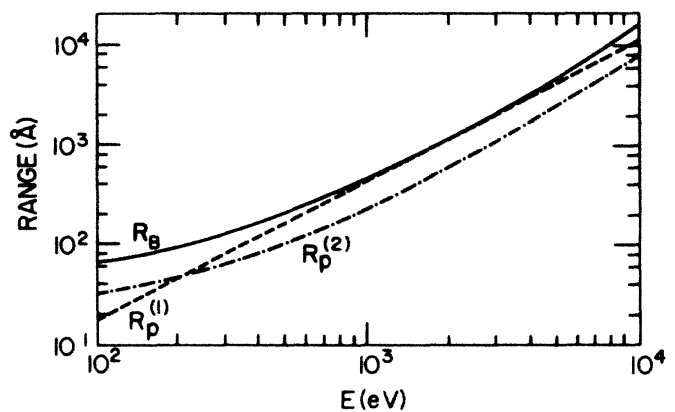

FIG. 8. Bethe range $R_{B}$ and penetration range estimates $R_{p}^{(1)}$ from Eq. (4) and $R_{p}^{(2)}$ from Eq. (5) for silicon.

inelastic mean free path $R_{\text {inel }}$ determines the sampling depth for EXAFS with detection of Auger electrons, which is much smaller than the sampling depth for EXAFS with total-electron-yield detection. ${ }^{37}$

Another aspect of total-electron-yield EXAFS is the production of large numbers of low-energy secondary electrons by $K L L$ and $L M M$ Auger electrons in cascades of inelastic collisions. Many secondary electrons created within an escape depth, on the order of $10-100 \AA,{ }^{38}$ of the specimen surface will reach the surface with sufficient kinetic energy to escape, and these constitute most of the observed total-electron-yield current. The minimum kinetic energy for escape by secondary electrons is determined by the work function. Work functions are typically in the range of $2-5 \mathrm{eV} .{ }^{39}$ Most secondary electrons which escape have kinetic energies of $3-5 \mathrm{eV} .40$

Predicting effects of silicon overlayers on the jump in total-electron-yield current at the Ge absorption edge requires an accurate treatment of the propagation and inelastic scattering of primary Auger electrons and of secondary electrons. Monte Carlo calculations have been used to model secondary electron production and propagation in electron lithography. ${ }^{41}$ However, we are aware of no Monte Carlo calculations which deal specifically with secondary electron yield from Auger processes. This would require following secondary electrons to lower kinetic energies than has been done in lithography studies. Available Monte Carlo calculations suggest simple approximations for interpreting the present total-electron-yield experiments on sample depth. (See Fig. 3 of Ref. 41, for example.)

\section{Simplifying assumptions}

We have developed an analytical model to describe edge jump versus overlayer thickness $t_{\mathrm{Si}}$ in terms of Auger electron penetration ranges $R_{p}$ and secondary electron escape lengths $\alpha^{-1}$, as defined in Eq. (8) below.

TABLE II. Penetration range estimates $R_{p}^{(1)}$ from Eq. (4) and $R_{p}^{(2)}$ from Eq. (5), Bethe range $R_{B}$, and inelastic mean free path $R_{\text {inel }}$ for $K L L$ and $L M M$ Auger electrons and for $0.5-\mathrm{keV}$ photoelectrons for several systems.

\begin{tabular}{|c|c|c|c|c|c|}
\hline System & $\begin{array}{c}\text { Auger energy } \\
(\mathrm{keV})\end{array}$ & $\begin{array}{l}R_{p}^{(1)} \\
(\AA) \\
\end{array}$ & $\begin{array}{l}R_{p}^{(2)} \\
(\AA) \\
\end{array}$ & $\begin{array}{l}R_{B} \\
(\AA) \\
\end{array}$ & $\begin{array}{c}R_{\text {inel }} \\
(\AA) \\
\end{array}$ \\
\hline \multicolumn{6}{|l|}{$\mathrm{Ge}-\mathrm{in}-\mathrm{Ge}$} \\
\hline$K L L$ & 8.6 & 3823 & 3850 & 7700 & 57 \\
\hline$L M M$ & 1.1 & 100 & 215 & 430 & 20 \\
\hline photoelectron & 0.5 & & 91 & 183 & 14 \\
\hline \multicolumn{6}{|l|}{$\mathrm{Ni}-\mathrm{in}-\mathrm{Ni}$} \\
\hline$K L L$ & 6.5 & 1544 & 1360 & 2700 & 35 \\
\hline$L M M$ & 0.85 & 89 & 85 & 170 & 12 \\
\hline photoelectron & 0.5 & & 52 & 105 & 10 \\
\hline \multicolumn{6}{|l|}{$\mathrm{Cu}$-in- $\mathrm{Cu}$} \\
\hline$K L L$ & 7.0 & 1705 & 1500 & 3000 & 37 \\
\hline$L M M$ & 0.92 & 100 & 95 & 190 & 13 \\
\hline photoelectron & 0.5 & & 51 & 102 & 10 \\
\hline \multicolumn{6}{|l|}{ Ge-in-Si } \\
\hline$K L L$ & 8.6 & 8720 & 6000 & 12000 & 54 \\
\hline$L M M$ & 1.1 & 490 & 275 & 550 & 19 \\
\hline photoelectron & 0.5 & & 103 & 207 & 13 \\
\hline \multicolumn{6}{|l|}{ As-in-Si } \\
\hline$K L L$ & 9.1 & 9467 & 6500 & 13000 & 56 \\
\hline$L M M$ & 1.2 & 554 & 300 & 600 & 20 \\
\hline photoelectron & 0.5 & & 103 & 207 & 13 \\
\hline \multicolumn{6}{|l|}{ Al-in- $\mathrm{Al}_{2} \mathrm{O}_{3}$} \\
\hline$K L L$ & 1.4 & 403 & 190 & 380 & 14 \\
\hline$L M M$ & 0.064 & & 15 & 31 & 3 \\
\hline photoelectron & 0.5 & & 56 & 113 & 8 \\
\hline
\end{tabular}


For simplicity, the subscript " $p$ " has been dropped from $R_{p}$ in the discussions and equations which follow in Secs. IV C and IV D. The model implicitly includes inelastic scattering and collision cascade phenomena, and it provides an estimate of sampling depth for totalelectron-yield EXAFS experiments. The model is based on five key assumptions.

(1) The total-electron-yield current for photon energies $E>E_{0}$ consists mainly of secondary electrons excited by Auger electrons.

(2) Through inelastic scattering and collision cascade processes, Auger electrons of range $R$ create secondary electrons uniformly throughout the volume of a sphere of radius $R$ centered on the source of the Auger electrons. (See Fig. 9 and, for example, Monte Carlo simulation results for secondary electron production in Ref. 41.)

(3) The velocity distribution for secondary electrons is isotropic, and the escape probability for secondary electrons created a distance $x$ away from the free surface is given by

$$
P(x)=\frac{1}{2}\left[e^{-\beta x}-\alpha x E_{1}(\beta x)\right],
$$

where $E_{1}(x)$ is the exponential integral

$$
-\operatorname{Ei}(-x)=\int_{x}^{\infty} \frac{e^{-v}}{v} d v
$$

Kanter $^{42}$ and Jones and Woodruff ${ }^{8}$ have derived similar expressions in related contexts. With $\exp (-\beta r)$ giving the probability of escape for an electron which travels a distance $r$ to reach the surface, $P(x)$ for the isotropic velocity distribution can be approximated for $x \beta \leq 1$ as

$$
P(x)=\frac{1}{2} e^{-\alpha x},
$$

with $\alpha \approx 2 \beta$.

(4) The secondary electron escape length $\alpha^{-1}$ is much less than the Auger electron range $R$, so $\alpha R \gg 1$.

(5) Each Auger electron of initial energy $E$ which remains within the solid eventually results in excitation of $N$ secondary electrons of average energy $\varepsilon$ whose escape probability is described by Eq. (6), and $N=E / \varepsilon$.

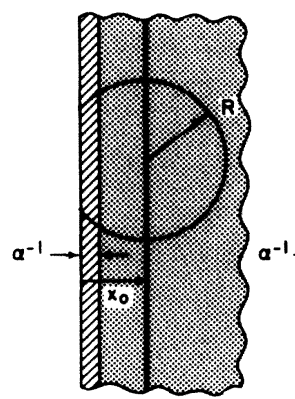

(a) $x_{0} \leq R$

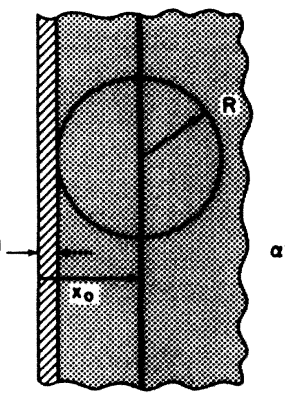

(b)

$x_{0} \geq R$

$x_{0} \approx R+a^{-1}$

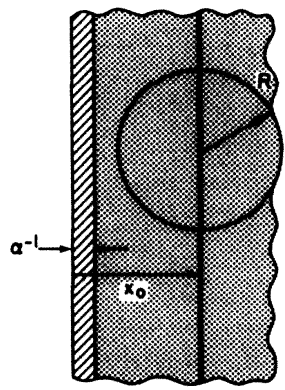

(c) $x_{0}>R$ $x_{0}>R+a^{-1}$
FIG. 9. Relationships between source depth $x_{0}$, Auger electron range $R$, and secondary electron escape length $\alpha^{-1}$, for the situations represented by Eqs. (9) and (10).

\section{Analytical model for total-electron-yield depth dependence}

As shown in Appendix B, these assumptions lead to the following general expressions for the contribution to the secondary-electron-yield current per Auger electron created at depth $x_{0}$ with range $R$ for a homogeneous planar sample:

$i\left(x_{0}\right)=\frac{3}{8} \frac{N}{\alpha R}\left[1-\left[\frac{x_{0}}{R}\right)^{2}+2 \frac{x_{0}}{R} \frac{1}{\alpha R}-\frac{2}{(\alpha R)^{2}}\right]$

for $x_{0} \leq R$ as pictured in Fig. 9(a), and

$i\left(x_{0}\right)=\frac{3}{8} \frac{N}{\alpha R}\left(\frac{2}{\alpha R}-\frac{2}{(\alpha R)^{2}}\right) \exp \left[-\alpha\left(x_{0}-R\right)\right]$

for $x_{0} \geq R$, as in Figs. 9(b) and 9(c). These expressions are plotted in Fig. 10 for $E=8.6$ and $1.1 \mathrm{keV}$, and $R=6000$ and $275 \AA$, which are $R_{p}^{(2)}$ values for germanium $K L L$ and $L M M$ Auger electrons in silicon, and with $\alpha^{-1}=5 \AA$ and $\varepsilon=8 \mathrm{eV}$. Since $R \gg \alpha^{-1}$, the depth sensitivity is determined primarily by the Auger electron penetration range $R$, with negligible secondary electron yield for $x_{0}>R$.

\section{E. Role of Auger electrons}

To describe the edge jump versus overlayer thickness for a Ge layer of thickness $t_{\mathrm{Ge}}$ covered by a Si layer of thickness $t_{\mathrm{Si}}$, it is necessary to consider both $K L L$ and $L M M$ Auger processes which result from $K$-shell absorption by $\mathrm{Ge}$ atoms. Relationships among relevant processes are illustrated in Fig. 7. More detailed analysis would include other, less probable Auger processes, ${ }^{21,43}$ e.g., $K L M$, without significantly changing the conclusions of this section.

For each X-ray-absorption event which creates a $\mathrm{Ge}$ $K$-shell hole, there are several processes by which primary and secondary electrons which constitute the total-electron-yield current may be emitted. Each $K$ shell absorption event produces one primary photoelectron and one $K$-shell hole. The numbers of $K L L$ and LMM Auger electrons which eventually result from decay of the hole depend on the radiative $\left(\omega_{K}, \omega_{L}\right)$ and

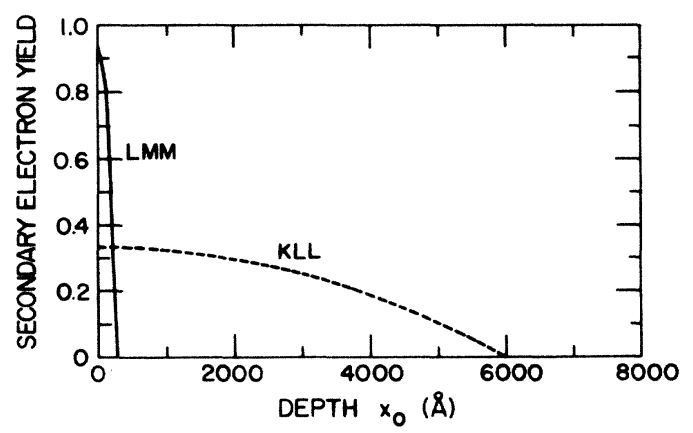

FIG. 10. Contributions to secondary-electron-yield current per Auger electron created at depth $x_{0}$ for Ge $K L L(E=8.6$ $\mathrm{keV}$ and $R=6000 \AA)$ and for $L M M(E=1.1 \mathrm{keV}$ and $R=275$ $\AA$ ) Auger electrons in Si with $\alpha^{-1}=5 \AA$ and $\varepsilon=8 \mathrm{eV}$. 
nonradiative (Auger) yields $\left(a_{K}, a_{L}\right)$ for $K$ and $L$ shells, ${ }^{44}$ and on relative emission rates, ${ }^{45} K_{\alpha} /\left(K_{\alpha}+K_{\beta}\right)$ and $K_{\beta} /\left(K_{\alpha}+K_{\beta}\right)$.

For the case of Ge-in-Si, the number of $K L L$ Auger electrons created for each Ge $K$-shell absorption event is given by

$$
n_{K L L}=a_{K}=0.47 \text {. }
$$

The number of LMM Auger electrons is given by

$$
n_{L M M}=n_{L M M}^{(1)}+n_{L M M}^{(2)}=1.38,
$$

where

$$
n_{L M M}^{(1)}=a_{K} \times 2 \times a_{L}=0.47 \times 2 \times 0.98=0.92
$$

is the number of the $L M M$ Auger electrons from nonradiative decay of the two $L$-shell holes produced by the $K L L$ Auger process, and

$n_{L M M}^{(2)}=\omega_{K} \frac{K_{\alpha}}{K_{\alpha}+K_{\beta}} a_{L}=0.53 \times 0.87 \times 0.98=0.45$

is the number of $L M M$ Auger electrons from nonradiative decay of the one $L$-shell hole produced by $K \alpha$ radiative decay of the original $K$-shell hole.

It follows that each $K$-shell absorption event's contribution to the total-electron-yield current is $i_{K L L}\left(x_{0}\right)$, as given by Eqs. (9) and (10) with

$$
N=n_{K L L} E_{K L L} / \varepsilon=505
$$

and $i_{L M M}\left(x_{0}\right)$ with

$$
N=n_{L M M} E_{L M M} / \varepsilon=1.90 \text {. }
$$

In both cases it has been assumed that $\varepsilon=8 \mathrm{eV}$.

\section{F. Role of photoelectrons}

The relative contributions from photoelectrons and from $K L L$ and $L M M$ Auger electrons to the secondary electron current for total-electron-yield EXAFS depend on the relative numbers of primary photoelectrons and Auger electrons which result from a core absorption event, as well as on the number of secondary electrons emitted per primary electron. Photoelectron contributions in total-electron-yield measurements were discussed in a preceding section (Sec. II A) with the conclusion that the photoelectron contribution to $\chi(k)$ is less than $10 \%$ of the total $\chi(k)$ up to $k=12 \AA^{-1}$, based on the experimental results of Fig. 3. In this section, the photoelectron contribution to the total-electron-yield current is evaluated using the model for total-electron-yield depth dependence, without reexamining whether or not the photoelectron-derived current contains EXAFS oscillations and contributes to $\chi(k)$.

The energy of photoelectrons produced in $x$-ray absorption depends on the difference between the incident photon energy $h v$ and the absorption threshold $E_{0}$. For each photoelectron of energy $E=h v-E_{0}$, Eqs. (9) and (10) can be used to estimate the resulting secondaryelectron-yield current. For $k=12 \AA^{-1}, E \approx 0.5 \mathrm{keV}$, and for $k=16 \AA^{-1}, E \approx 1 \mathrm{keV}$, which are less than the ener- gies of $K L L$ Auger electrons for the systems in Table II. The photoelectrons are expected to have correspondingly smaller ranges than the $K L L$ Auger electrons (see Fig. 8 and Table II).

For $K$-shell absorption events for $\mathrm{Ge}$-in-Si, the photoelectron contribution $i_{\text {photoel }}\left(x_{0}\right)$ to the total-electronyield current depends upon the initial energy of the photoelectron, $E_{\text {photoel }}$, and is given by Eqs. (9) and (10) with

$$
N=E_{\text {photoel }} / \varepsilon=62
$$

for $k=12 \AA^{-1}$ and taking $\varepsilon=8 \mathrm{eV}$.

The dependence of $i_{K L L}, i_{L M M}$, and $i_{\text {photoel }}$ on silicon overlayer thickness $t_{\mathrm{Si}}$ can be calculated from Eqs. (9) and (10) with $x_{0}=t_{\mathrm{Si}}$, with the various substitutions for $N$ given above, and with range values of $R_{p}^{(2)}$ from Table II for $R_{K L L}, R_{L M M}$, and $R_{\text {photoel }}$. The results are shown in Fig. 11. The dependence of edge-jump current on $t_{\mathrm{Si}}$, Fig. 6, does not involve $i_{\text {photoel }}$, since the photoelectron energy approaches zero at the edge.

Note that an overlayer of $500 \AA$ of Si should eliminate contributions from $i_{L M M}$ and from $i_{\text {photoel }}$, with little effect on $i_{K L L}$. In some situations it may be desirable to use overlayers to eliminate the great very-near-surface sensitivity which may otherwise result from $L M M$ and photoelectrons in total-electron-yield EXAFS measurements. For example, see Sec. III B and Figs. 3-5.

\section{G. Other total-electron-yield detection methods}

Conclusions on sampling depth must be reevaluated for other types of total-electron-yield detectors: gas-flow electron detectors operated in ion chamber mode $^{10,12,14-16}$ and electron-multiplier-type detectors operated in pulse mode. ${ }^{5-9}$

For a gas-flow detector employing helium, only ejected electrons with kinetic energy at least as large as the ionization potential of helium, $24.5 \mathrm{eV}$, contribute to the detector current, unless the detector is operated at low gas pressure and high applied voltage in the proportional regime rather than in the more usual ion chamber regime. In the latter case, most of the secondary electrons, which have energies less than $20 \mathrm{eV}$, are not detected, although they constitute most of the total-

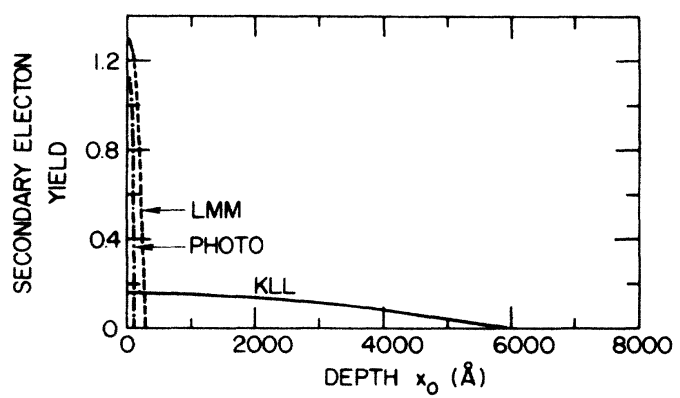

FIG. 11. Contributions to secondary-electron-yield current per Ge $K$-shell absorption event at depth $x_{0}$ for Ge $K L L(8.6$ $\mathrm{keV})$ and $L M M(1.1 \mathrm{keV})$ Auger electrons and for $0.5-\mathrm{keV}$ photoelectrons in Si with $\alpha^{-1}=5 \AA, \varepsilon=8 \mathrm{eV}$, and values of $R_{p}^{(2)}$ from Table II. 
electron-yield current for the specimen-current-type measurements described in Sec. II. Also, higher-energy ejected electrons may cause multiple ionization events, each contributing to the output of the gas-flow detector, which is not true for the specimen current measurements. For these reasons, assumption (1) of Sec. IV C is not applicable for gas-flow detection, where ejected Auger electrons and fast secondary electrons are more important than low-energy secondary electrons. This should lead to a smaller sampling depth for gas-flow detection than for specimen-current-type measurements, ${ }^{16}$ e.g., smaller sampling depths than given by values of $R_{p}$ from Eqs. (4) and (5) and from Table II. Bouldin et al. ${ }^{16}$ have reported a sampling depth of 700-1000 $\AA$ for gas-flow detection of $\mathrm{Ga}$ and As $K$-edge EXAFS for GaAs. For this case, $R_{p}^{(2)}$ values for $K L L$ Auger electrons are 3420-3600 ̊.

For the case of electron-multiplier-type detectors operated in a pulse mode with synchrotron radiation, a binary response, either no pulse or one pulse from the detector, occurs for each burst of incident photons. The linearity of this type of measurement depends upon having a sufficiently low probability of detected electron emission for any single burst of incident radiation. For an electron multiplier which is biased positive with respect to the specimen, a single secondary electron, Auger electron, or photoelectron may trigger the detector. The depth sensitivity and linearity of such measurements should depend on the per-burst incident energy, the nature of the sample, and the operating characteristics of the electron multiplier. Comparisons between depth sensitivity calculations using the model of Sec. IV $D$ and measurements ${ }^{8}$ made with an electron multiplier detector are given in Secs. V B and V C.

\section{COMPARISONS OF CALCULATIONS WITH EXPERIMENTAL RESULTS}

\section{A. Germanium in silicon}

Modeling the edge-jump total-electron-yield current versus overlayer thickness for the Ge-in-Si experiment requires special consideration of the $t_{\mathrm{Si}}=0$ situation, because of the finite, $100-\AA$ thickness of the Ge layer, throughout which $K$-shell absorption occurs. The various contributions to the total-electron-yield current for $t_{\mathrm{Si}}=0$ are given by integrating Eq. (9) from $x_{0}=0$ to $x_{0}=100 \AA$ using the appropriate values of $R$ for $K L L$ and $L M M$ Auger electrons propagating in $\mathrm{Ge}$.

The results obtained with Eqs. (9)-(17) for the case of Ge-in-Si are shown in Fig. 6, together with the measured values of edge jump versus overlayer thickness. The calculation employed $R_{p}^{(2)}$ ranges for $K L L$ and $L M M$ Auger electrons and took $\varepsilon=8 \mathrm{eV}$.

For these absolute comparisons between calculated and observed edge-jump total-electron-yield currents, the incident $x$-ray flux was estimated from the output of the nitrogen-filled (1 atm) ionization chamber used to monitor $I_{0}$ (see Fig. 1), assuming $30 \mathrm{eV}$ of absorbed x-ray energy for each collected electron-ion pair. Absorption coefficients were taken from Ref. 46 for nitrogen and germanium.

For the $t_{\mathrm{Si}}=0$ sample, the time-integrated edge-jump current was $1.6 \times 10^{-12} \mathrm{~A} \mathrm{sec}$, or $9.9 \times 10^{6}$ electrons, for $10^{10}$ incident photons, or $8.9 \times 10^{6} \mathrm{Ge} K$-shell absorption events, giving 1.1 total-electron-yield electrons per absorption event. This dropped to 0.09 total-electron-yield electrons per absorption event for $t_{\mathrm{Si}}=4000 \AA$, as shown in Fig. 6(b).

The best agreement between observed and calculated total-electron-yield currents for $t_{\mathrm{Si}}=2000-4000 \AA$ corresponds to $\alpha^{-1}=5 \AA$ with $\varepsilon=8 \mathrm{eV}$, although only the product $\alpha \varepsilon=1.6 \AA^{-1} \mathrm{eV}$ can be determined in this way, and a factor of 2 uncertainty in the incident $x$-ray flux and in Auger electron ranges make the $\alpha \varepsilon$ values only order-of-magnitude estimates.

\section{B. Copper on nickel}

Martens et $a l .{ }^{6}$ reported the dependence of the $\mathrm{Cu} K$ edge jump as a function of $\mathrm{Cu}$ layer thickness on a $\mathrm{Ni}$ substrate, as shown in Fig. 12. These measurements were obtained with an electron-multiplier-type detector. Also shown in Fig. 12 is the behavior expected from

$$
i(t)=\int_{0}^{t} i(x) d x
$$

with $i(x)$ from Eq. (9) and $R_{p}^{(2)}$ values from Table II for $K L L$ and $L M M \mathrm{Cu}$ Auger electrons. $N_{K L L}$ and $N_{L M M}$ were calculated as outlined in Sec. IV E. Results of this calculation agree very well with the experimental observations.

\section{Aluminum oxide on aluminum}

Estimates of sampling depths for total-electron-yield detection were reported by Jones and Woodruff ${ }^{8}$ for aluminum oxide films of up to $400-\AA$ thickness. They used an electron-multiplier-type detector in a pulse mode and measured the EXAFS signal strength from aluminum oxide overlayers on bulk aluminum. For comparison with the experimental results of Jones and Woodruff, ${ }^{8}$ calculations of the $\mathrm{Al} K$-edge jump for $\mathrm{Al}_{2} \mathrm{O}_{3}$ were carried out like those for $\mathrm{Cu}$ on $\mathrm{Ni}$. As shown in Fig. 13, results from these calculations agree fairly well with the experimental observations. Differences may be

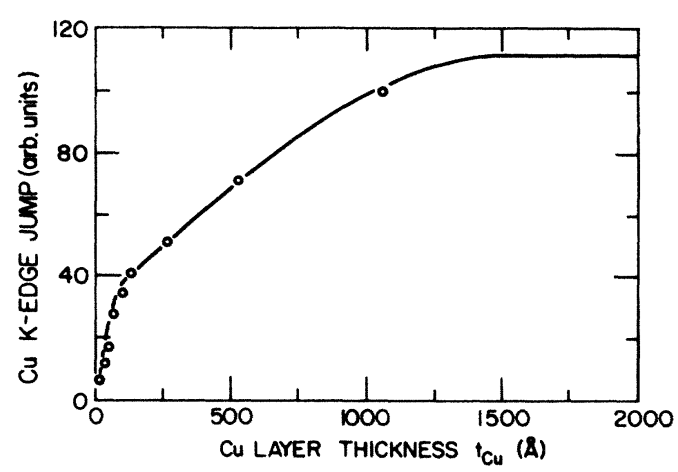

FIG. 12. Observed $\mathrm{Cu} K$-edge jump vs $\mathrm{Cu}$ layer thickness from Martens et al. (Ref. 6) (data points) and calculated edge jump vs layer thickness from Eq. (18). 


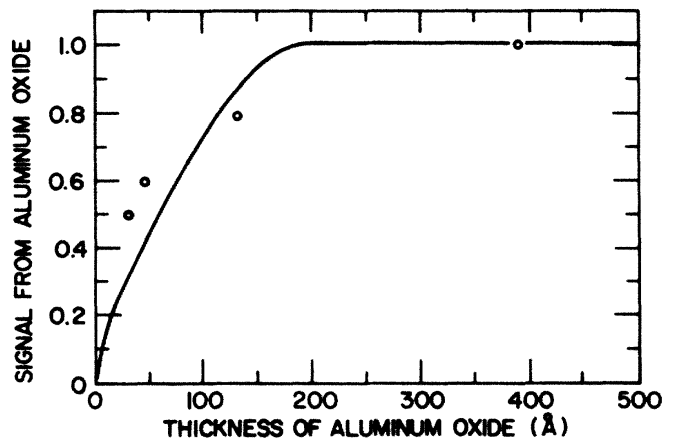

FIG. 13. Observed EXAFS signal strength from an aluminum oxide layer relative to that from underlying bulk aluminum from Ref. 8 (data points) and calculated signal strength from Eq. (18).

due in part to uncertainties in the actual overlayer thicknesses in the experiments and in the value of $R_{p}^{(2)}$ used for $L M M$ Auger electrons in the calculation. Also, the experimental data are the fraction of the EXAFS signal due to the oxide relative to the total EXAFS signal from oxide and from bulk aluminum, and the calculation is for the change of edge-jump current from the oxide with oxide thickness.

\section{CONCLUSIONS}

The work reported in this paper demonstrates that direct measurement of specimen current can yield accurate EXAFS $\chi(k)$ functions, if appropriate cautions are observed. Problems from different structural environments in very-near-surface and in deeper, subsurface regions and problems from contributions to the specimen current from electrons which exit from the rear surface of thin samples must be avoided. Experiments confirm that the sampling depth for total-electron-yield, specimen-current EXAFS measurements is determined by the penetration ranges of Auger electrons emitted from the absorbing atoms. Expressions derived for the sampling depth are verified by comparisons with experimental data for several different materials.

\section{ACKNOWLEDGMENTS}

We thank C. Jahnes for preparing the Si-Ge-Si samples, P. Saunders for help with backscattering analysis, O. C. Wells for useful discussions of electron penetration ranges, and the staff of CHESS for help with EXAFS measurements.

\section{APPENDIX A: REAR-SURFACE CONTRIBUTIONS IN TOTAL-ELECTRON-YIELD EXAFS}

The following is an analysis of the effect of including in the total-electron-yield current those electrons which exit from the rear surface of a thin-foil sample. As noted in Sec. III, EXAFS $\chi(k)$ functions from totalelectron-yield measurements can have significantly reduced amplitudes when rear-surface contributions are in- cluded. An expression is derived which relates the correct $\chi(k)$ function, which would result from totalelectron-yield measurements which include only electrons from the front surface, and the incorrect $\chi(k)$ function, denoted $\chi_{\text {tot }}(k)$, which includes both the frontand rear-surface contributions. This expression involves the thickness $t$ of the sample; the x-ray-absorption coefficient for $K$-shell events, smoothed to remove EXAFS oscillations, $\mu_{K, 0}$; and the absorption coefficient for non- $K$-shell events, $\mu_{\text {non- } K}$. The relationships among $\mu$, $\mu_{K}, \mu_{K, 0}, \mu_{\text {non- } K}$, and $\chi(k)$ have already been given in Eqs. (1) and (2).

Consider the situation shown in Fig. 14 for a planar sample of thickness $t$ perpendicular to the incident $\mathrm{x}$-ray beam of flux $I_{0}(E)$, with transmitted x-ray flux $I_{t}(E)$, and with total-electron-yield current $i_{\text {tot }}(E)$ produced by absorption of $\mathrm{x}$ rays of energy $E . I_{0}(z, E)$ is the $\mathrm{x}$-ray flux at depth $z(z \leq t)$ within the sample, $I_{0}(z, E) \mu(E) d z$ is the flux lost by absorption in a slice of thickness $d z$ at depth $z$, and

$$
I_{0}(z, E)=I_{0}(E) \exp [-z \mu(E)] .
$$

The following approximations are introduced to simplify the analysis.

(1) Consider only one type of Auger electron, e.g., those from $K L L$ processes, and represent the Auger yield, the number of Auger electrons produced per $K$ shell absorption event, by $a$. Each Auger electron creates $N$ secondary electrons.

(2) A fixed fraction $\eta$ of the secondary electrons created as a result of absorption events within a distance $\lambda$ of each surface, and only these, escape. (At this level of approximation, $\lambda$ can be considered to be the Auger electron range.)

(3) The contribution to the front-surface totalelectron-yield current from non- $K$-shell absorption events is given by $\zeta I_{0}(E) \mu_{\text {non- } K}(E)$ and the contribution to the rear-surface current is $\zeta I_{0}(E) \exp \left[-t \mu_{\mathrm{tot}}(E)\right]$ $\times \mu_{\text {non- } K}(E)$, with the same constant $\zeta$.

(4) The $x$-ray-absorption length $\mu^{-1}$ is much greater than $\lambda$.

(5) The sample thickness $t$ is much greater than $\lambda$ but

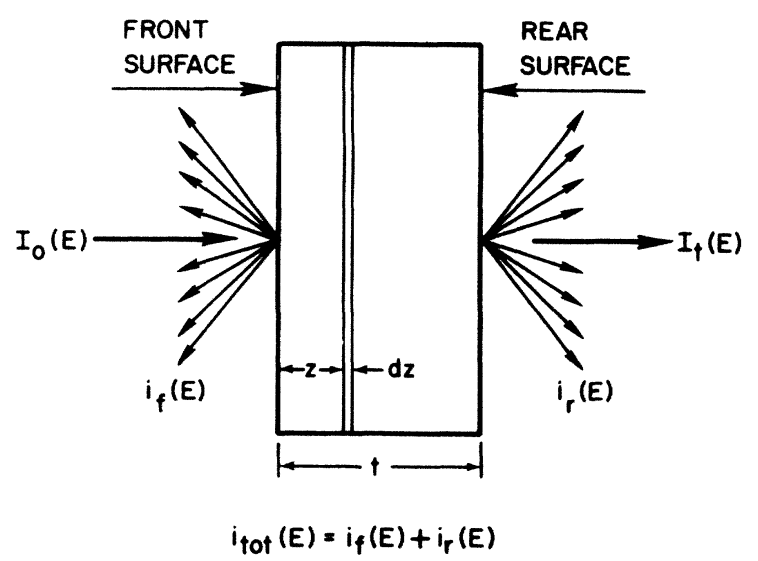

FIG. 14. Planar specimen with total-electron-yield current collection from front and rear surfaces, as described in the text. 
on the order of the $\mathrm{x}$-ray-absorption length $\mu^{-1}$.

The total current $i_{\text {tot }}(E)$ is the sum of the frontsurface current $i_{f}(E)$ and the rear-surface current $i_{r}(E)$. For energies above the absorption edge, $E>E_{0}$, these two components of the total-electron-yield current (number of escaping secondary electrons) can be written

$$
i_{f}(E)=I_{0}(E)\left[\lambda a N \eta \mu_{K}(E)+\zeta \mu_{\text {non- } K}(E)\right]
$$

and

$$
i_{r}(E)=i_{f}(E) \exp [-t \mu(E)] .
$$

For $E<E_{0}$,

$$
i_{f}(E)=I_{0}(E) \xi \mu_{\text {non-K }}(E)
$$

and $i_{r}(E)$ is again given by Eq. (A3).

Proceeding to calculate $\chi(k)$ functions from the totalelectron-yield current, as outlined in Sec. III,

$$
\begin{aligned}
& i_{K, f}(E)=I_{0}(E) \lambda a N \eta \mu_{K}(E), \\
& i_{K, 0, f}(E)=I_{0}(E) \lambda a N \eta \mu_{K, 0}(E),
\end{aligned}
$$

and

$$
\begin{aligned}
& i_{K, r}(E)=I_{0}(E) \lambda a N \eta \mu_{K}(E) \exp [-t \mu(E)], \\
& i_{K, 0, r}(E)=I_{0}(E) \lambda a N \eta \mu_{K, 0}(E) \exp [-t \mu(E)] .
\end{aligned}
$$

It follows that

$$
\begin{aligned}
\chi_{f}(E) & =\frac{i_{K, f}(E)-i_{K, 0, f}(E)}{i_{K, 0, f}\left(E_{0}\right)} \\
& =\frac{\lambda N \eta\left[\mu_{K}(E)-\mu_{K, 0}(E)\right]}{\lambda N \eta \mu_{K, 0}\left(E_{0}\right)}=\chi(E)
\end{aligned}
$$

and

$$
\begin{aligned}
\chi_{\mathrm{tot}}(E) & =\frac{i_{K, \text { tot }}(E)-i_{K, 0, \text { tot }}(E)}{i_{K, 0, \text { tot }}\left(E_{0}\right)} \\
& =\frac{\mu_{K}-\mu_{K, 0}+\exp \left(-t \mu_{\text {non }-K}\right)\left[\mu_{K} \exp \left(-t \mu_{K}\right)-\mu_{K, 0} \exp \left(-t \mu_{K, 0}\right)\right]}{\mu_{K, 0}\left\{1+\exp \left[-t\left(\mu_{\text {non }-K}+\mu_{K, 0}\right)\right]\right\}} .
\end{aligned}
$$

Equation (A10) can be rewritten as

$$
\begin{aligned}
\chi_{\mathrm{tot}}(E)= & \frac{\chi}{1+\exp \left[-t\left(\mu_{\text {non- } K}+\mu_{K, 0}\right)\right]} \\
& +\frac{\exp \left(-t \mu_{\text {non }-K}\right)}{1+\exp \left[-t\left(\mu_{\text {non }-K}+\mu_{K, 0}\right)\right]} \exp \left(-t \mu_{K, 0}\right)\left[\chi \exp \left(-t \mu_{K, 0} \chi\right)+\exp \left(-t \mu_{K, 0} \chi\right)-1\right] .
\end{aligned}
$$

With the approximation $\exp \left(-t \mu_{K, 0} \chi\right) \approx 1-t \mu_{K, 0} \chi$, Eq. (A11) can be simplified to the following:

$$
\begin{aligned}
& \chi_{\mathrm{tot}}(E)= \chi(E) \frac{1}{1+\exp \left[-t\left(\mu_{\mathrm{non}-K}+\mu_{K, 0}\right)\right]} \\
& \times\left\{1+\left(1-t \mu_{K, 0}\right)\right. \\
& \\
&\left.\quad \times \exp \left[-t\left(\mu_{\text {non }-K}+\mu_{K, 0}\right)\right]\right\} .
\end{aligned}
$$

In the limit $t\left(\mu_{\text {non- } K}+\mu_{K, 0}\right) \gg 1$, Eq. (A12) reduces to $\chi_{\text {tot }}(E)=\chi(E)$ as expected, since there is no rear-surface current in this thick sample limit. In the thin sample limit, Eq. (A11) reduces to

$$
\chi_{\text {tot }}(E)=\chi(E)\left(1-\frac{t \mu_{K, 0}}{2}\right) \text {. }
$$

A series of plots for $\chi_{\text {tot }} / \chi$ versus $t \mu_{K, 0}$ are shown in Fig. 15 for different values of $\mu_{\text {non- } K} / \mu_{K, 0}$, calculated using Eq. (A12). Also shown in Fig. 15 is the thin sample limit, Eq. (A13). For nickel at the $K$ edge, $\mu_{\text {non- } K} / \mu_{K, 0}=0.15$; for cobalt, $0.14 .^{46}$ The two data points in Fig. 15 are from total-electron-yield current measurements on $5-\mu \mathrm{m}$-thick films of nickel and cobalt, which are listed in Table $\mathrm{I}$ as $\mathrm{Co}$ and $\mathrm{Ni}-1$. The observed amplitudes for $\chi_{\text {tot }}$ with respect to $\chi$ for these two samples, $\chi_{\text {tot }} / \chi=0.83$ and 0.82 , are approximately $10 \%$ larger than the values expected from Fig. 15. $\chi$ is taken from the total-electron-yield current measurements with a $100-\mu \mathrm{m}$-thick lead foil placed immediately behind the specimen and the metal frame (see Fig. 1), and $\chi_{\text {tot }}$ is taken from total-electron-yield measurements without the lead foil. The difference between observed and cal-

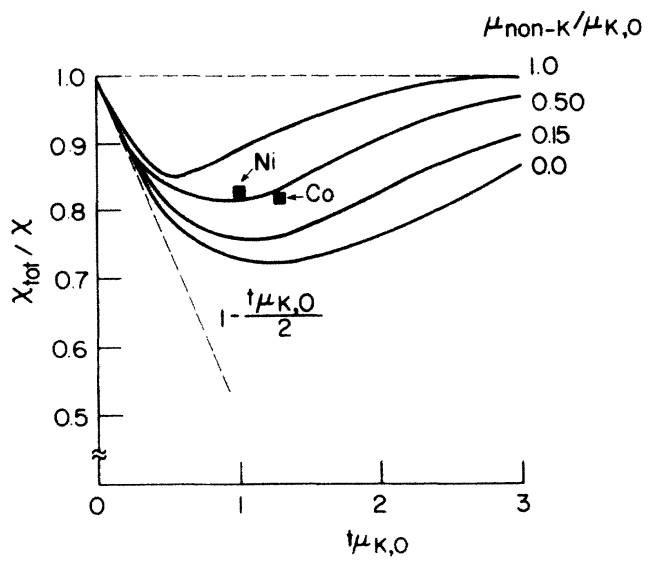

FIG. 15. Reduced EXAFS amplitudes in total-electron-yield measurements expected from including rear-surface contributions. The two data points are from measurements on $5-\mu \mathrm{m}$ thick nickel and cobalt foils. 
culated values of $\chi_{\text {tot }} / \chi$ may result in part from some escape of total-electron-yield electrons from the rear surface even with the lead foil in place, to introduce a part of the $i_{r}(E)$ current into $\chi(E) ; 30 \%$ leakage would be required to explain the entire difference. The surface oxides noted in Sec. III B, which cause reduced amplitudes for front-surface total-electron-yield measurements for samples $\mathrm{Co}$ and $\mathrm{Ni}-1$, may also affect the observed values of $\chi_{\text {tot }} / \chi$ for these samples.

\section{APPENDIX B: SECONDARY ELECTRON CURRENT IN TOTAL-ELECTRON-YIELD EXAFS}

The following derivation yields Eqs. (9) and (10) of Sec. IV D for the contribution to the secondaryelectron-yield current per Auger electron created at depth $x_{0}$ with range $R$ for a homogeneous planar sample. In Fig. 16(a) let the $(0, y, z)$ plane be the surface of the sample, which occupies the $x>0$ half-space. Consider Auger electrons of energy $E$ created at position $\mathbf{r}_{0}$ by



(a)

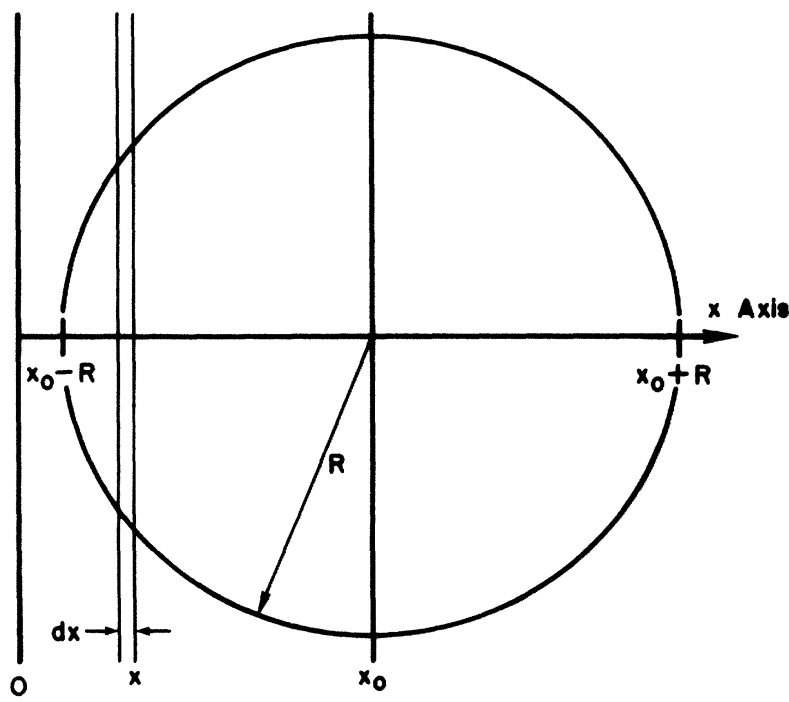

(b)

FIG. 16. Coordinate system and variables used in deriving expressions for secondary-electron-yield current. an x-ray-absorption event. The number of secondary electrons produced in volume element $d \mathbf{r}$ located at $\mathbf{r}$ by inelastic scattering per Auger electron originating from $\mathbf{r}_{0}$ is given by $n\left(\mathbf{r}, \mathbf{r}_{0}, E\right) d \mathbf{r}$. The probability that a secondary electron produced at $\mathbf{r}$ arrives at the sample surface $(0, y, z)$ and escapes into the vacuum is represented by $P(\mathbf{r})=P(x)$.

Following the approach used by Lye and Dekker, ${ }^{47}$ the number of secondary electrons which escape per Auger electron created at $\mathbf{r}_{0}$ with energy $E$ is given by

$$
i\left(\mathbf{r}_{0}\right)=i\left(x_{0}\right)=\int_{x>0} n\left(\mathbf{r}, \mathbf{r}_{0}, E\right) P(\mathbf{r}) d \mathbf{r} .
$$

To evaluate $n\left(\mathbf{r}, \mathbf{r}_{0}, E\right)$, assume that Auger electrons of range $R$ create secondary electrons uniformly throughout the volume of a sphere of radius $R$ centered on the source of the Auger electrons,

$n\left(\mathbf{r}, \mathbf{r}_{0}, E\right)=\left\{\begin{array}{l}n(E) \text { for } x \geq 0 \text { and }\left|\mathbf{r}-\mathbf{r}_{0}\right| \leq R(E), \\ 0 \text { for } x<0 \text { or }\left|\mathbf{r}-\mathbf{r}_{0}\right|>R(E) .\end{array}\right.$

For the case of $x_{0}>R$, the total number of secondary electrons created per Auger electron is given by

$N(E)=\int n\left(\left|\mathbf{r}-\mathbf{r}_{0}\right|, E\right) d \mathbf{r}=n(E) \frac{4}{3} \pi R^{3}=\frac{E}{\varepsilon}$,

where $\varepsilon$ is on the order of secondary electron energies.

For $\boldsymbol{P}(\mathbf{r})$, the escape probability for secondary electrons created at depth $x$ from the surface, Eq. (8) is used:

$$
P(\mathbf{r})=P(x)=\frac{1}{2} \exp (-\alpha x),
$$

where $\alpha^{-1}$ is the secondary electron escape length, which is much less than the Auger electron range $R$ for the cases of interest.

To evaluate $i\left(x_{0}\right)$, consider Fig. 16(b) and first calculate $n\left(x, x_{0}\right)$, the number of secondary electrons produced in the volume defined by the planes perpendicular to the $x$ axis at $x$ and $x+d x$ per Auger electron created at $\left(x_{0}, 0,0\right)$,

$$
n\left(x, x_{0}\right)= \begin{cases}\frac{3}{4} \frac{N}{R}\left[1-\left(\frac{x-x_{0}}{R}\right)^{2}\right] d x & \text { for }\left|x-x_{0}\right| \leq R, \\ 0 \text { for }\left|x-x_{0}\right|>R & \end{cases}
$$

and their contribution to the secondary electron yield,

$$
\begin{aligned}
i\left(x, x_{0}\right) d x & =n\left(x, x_{0}\right) P(x) d x \\
& =\frac{3}{4} N_{R}\left[1-\left[\frac{x-x_{0}}{R}\right]^{2}\right] \exp (-\alpha x) d x \\
& \text { for }\left|x_{0}-x\right| \leq R .
\end{aligned}
$$

The total contribution to the secondary electron current per Auger electron created at $\left(x_{0}, 0,0\right)$ is

$$
i\left(x_{0}\right)=\int_{\max \left\{0, x_{0}-R\right\}}^{x_{0}+R} n\left(x, x_{0}\right) P(x) d x .
$$

For $x_{0}<R$, this gives 


$$
\begin{aligned}
i\left(x_{0}\right)= & \frac{3}{8} \frac{N}{\alpha R}\left[1-\left(\frac{x_{0}}{R}\right)^{2}+2 \frac{x_{0}}{R} \frac{1}{\alpha R}-\frac{2}{(\alpha R)^{2}}\right] \\
& +\frac{3}{4} \frac{N}{\alpha R} \exp \left[-\alpha\left(R+x_{0}\right)\right]\left(\frac{1}{\alpha R}+\frac{1}{(\alpha R)^{2}}\right),
\end{aligned}
$$

(B8)

and for $x_{0}>R$,

$$
\begin{aligned}
i\left(x_{0}\right)= & \frac{3}{8} \frac{N}{\alpha R}\left[\frac{2}{\alpha R}-\frac{2}{(\alpha R)^{2}}\right) \exp \left[-\alpha\left(x_{0}-R\right)\right] \\
& +\frac{3}{4} \frac{N}{\alpha R} \exp \left[-\alpha\left(R+x_{0}\right)\right]\left(\frac{1}{\alpha R}+\frac{1}{(\alpha R)^{2}}\right) .
\end{aligned}
$$

In both Eqs. (B8) and (B9) the second term, containing $\exp \left[-\alpha\left(R+x_{0}\right)\right]$, can be neglected, since $\alpha R \gg 1$.
*Present address: Georgia Institute of Technology, School of Physics, Atlanta, GA 30332.

†Present address: HASYLAB at DESY, Notkestr. 85, 2000 Hamburg 52, West Germany.

${ }^{1}$ P. A. Lee, P. H. Citrin, P. Eisenberger, and B. M. Kincaid, Rev. Mod. Phys. 35, 769 (1981).

${ }^{2}$ T. M. Hayes and J. B. Boyce, in Solid State Physics, edited by H. Ehrenreich, F. Seitz, and D. Turnbull (Academic, New York, 1982), Vol. 37, p. 173.

3J. Jaklevic, J. A. Kirby, M. P. Klein, A. S. Robertson, G. S. Brown, and P. Eisenberger, Solid State Commun. 23, 679 (1977).

${ }^{4}$ P. A. Lee, Phys. Rev. B 13, 5261 (1976).

${ }^{5}$ P. H. Citrin, P. Eisenberger, and R. C. Hewitt, Phys. Rev. Lett. 41, 309 (1978); Surf. Sci. 89, 28 (1979); Phys. Rev. Lett. 45, 1948 (1980).

${ }^{6}$ G. Martens, P. Rabe, G. Tolkiehn, and A. Werner, Phys. Status Solidi A 55, 105 (1979).

${ }^{7}$ J. Stöhr, R. Jaeger, and S. Brennan, Surf. Sci. 11, 503 (1982).

${ }^{8}$ R. G. Jones and D. P. Woodruff, Surf. Sci. 114, 38 (1982).

${ }^{9}$ J. Stöhr, C. Noguera, and T. Kendelewicz, Phys. Rev. B 30, 5571 (1984).

${ }^{10}$ M. E. Kordesch and R. W. Hoffman, Phys. Rev. B 29, 491 (1984).

${ }^{11}$ A. Erbil, G. S. Cargill III, and R. F. Boehme, MRS Symp. Proc. 41, 275 (1985).

${ }^{12}$ T. Guo and M. L. denBoer, Phys. Rev. B 31, 6233 (1985).

${ }^{13}$ A. Erbil, W. Weber, G. S. Cargill III, and R. F. Boehme, Phys. Rev. B 34, 1392 (1986).

${ }^{14}$ K. Pandya, C. Natarajan, R. W. Hoffman, W. E. O'Grady, and D. E. Sayers, Brookhaven National Laboratory Report No. BNL-51947, UC-13, 1985 (unpublished).

${ }^{15}$ F. W. Lytle, R. B. Greegor, G. P. Huffman, and F. E. Huggins, Stanford Synchrotron Radiation Laboratory Report No. 86/01, 1985 (unpublished).

${ }^{16}$ C. E. Bouldin, R. A. Forman, and M. I. Bell, Phys. Rev. B 35, 1429 (1987).

${ }^{17}$ E. A. Stern and S. M. Heald, Rev. Sci. Instrum. 50, 1579 (1979).

18J. A. Golovchenko, B. W. Batterman, and W. L. Brown, Phys. Rev. B 10, 4239 (1974).

${ }^{19}$ M. V. Kruglov, V. N. Shchevelev, and G. G. Kareva, Phys. Status Solidi A 46, 343 (1978).

${ }^{20}$ T. Ohta, H. Sekiyama, Y. Kitajima, H. Kuroda, T. Takahashi, and S. Kikuta, Jpn. J. Appl. Phys. 24, L475 (1985).

${ }^{21}$ See, for example, T. A. Carlson, Photoelectron and Auger Spectroscopy (Plenum, New York, 1975), Chap. 6; R. L. Park, Solid State Physics: Surfaces, Vol. 22 of Methods of
Experimental Physics (Academic, Orlando, 1985), Chap. 4.

${ }^{22}$ See O. C. Wells, Scanning Electron Microscopy (McGrawHill, New York, (1974), p. 51, and references cited there.

${ }^{23}$ A. G. Grün, Z. Naturforsch. Teil A 12, 89 (1987).

${ }^{24}$ J. E. Holliday and E. J. Sternglass, J. Appl. Phys. 30, 1428 (1959).

${ }^{25}$ H. Kanter, Phys. Rev. 121, 461 (1961).

${ }^{26}$ H. Kanter and E. J. Sternglass, Phys. Rev. 126, 620 (1962).

${ }^{27}$ V. E. Cosslett and R. N. Thomas, Br. J. Appl. Phys. 15, 1283 (1964).

${ }^{28}$ H. Drescher, L. Reimer, and H. Seidel, Z. Angew. Phys. 29, 331 (1970).

${ }^{29}$ L. Reimer, Scanning Electron Microsc. II, 111 (1979).

${ }^{30}$ S. A. Rishton, S. P. Beaumont, and C. D. W. Wilkinson (unpublished).

${ }^{31}$ M. J. Berger and S. M. Seltzer, NASA Report No. SP-3112, 1964 (unpublished); NASA Report No. SP-3136, 1966 (unpublished) (available from National Technical Information Service, U.S. Department of Commerce, Springfield, VA 22161).

${ }^{32}$ H. Sugiyama, Jpn. Bull. Electrotech. Lab. 38, 351 (1974).

${ }^{33}$ D. Liljequist, T. Ekdahl, and U. Bäverstam, Nucl. Instrum. Methods 155, 529 (1978).

${ }^{34}$ A. Neukermans and W. Saperstein, J. Vac. Sci. Technol. 16, 1847 (1979).

${ }^{35}$ I. Lindau and W. E. Spicer, J. Electron Spectrosc. Relat. Phenom. 3, 409 (1974).

${ }^{36}$ M. P. Seah and W. A. Dench, Surf. Interface Anal. 1, 2 (1979).

${ }^{37}$ F. Comin, L. Incoccia, P. Lagarde, G. Rossi, and P. H. Citrin, Phys. Rev. Lett. 54, 122 (1985).

${ }^{38}$ H. Seiler, Z. Angew. Phys. 22, 249 (1967).

${ }^{39}$ J. C. Riviere, Solid State Surf. Sci. 1, 179 (1969).

${ }^{40} \mathrm{R}$. Kollath, in Electron-Emission Gas Discharges I, Vol. 21 of Handbuch der Physik (Springer-Verlag, Berlin, 1956), p. 232.

${ }^{41}$ K. Murata, D. F. Kyser, and C. H. Ting, J. Appl. Phys. 52, 4396 (1981).

${ }^{42}$ H. Kanter, Phys. Rev. B 1, 522 (1970).

${ }^{43}$ P. Erman, I. Bergström, Y. Y. Chu, and G. T. Emery, Nucl. Phys. 62, 401 (1975).

${ }^{44}$ M. O. Krause, J. Phys Chem. Ref. Data 8, 307 (1979).

${ }^{45}$ S. I. Salem, S. L. Panossian, and R. A. Krause, At. Data Nucl. Data Tables 14, 91 (1974).

${ }^{46}$ W. H. McMaster, N. Kerr Del Grande, J. H. Mallett, and J. H. Hubbell, Lawrence Radiation Laboratory Report No. UCRL-50174-SEC.2 R-1, 1969 (unpublished) (available from National Technical Information Service, U.S. Department of Commerce, Springfield, VA 22161).

${ }^{47}$ R. G. Lye and A. J. Dekker, Phys. Rev. 107, 977 (1957). 

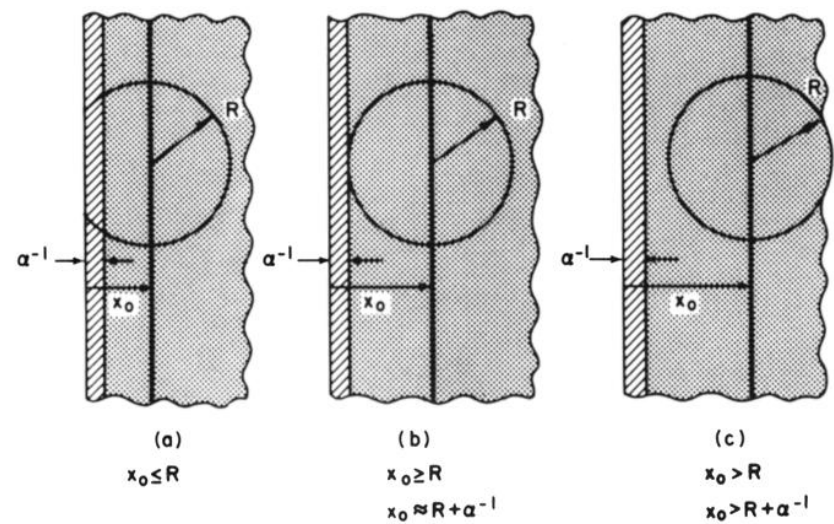

FIG. 9. Relationships between source depth $x_{0}$, Auger electron range $R$, and secondary electron escape length $\alpha^{-1}$, for the situations represented by Eqs. (9) and (10). 